\title{
ON SCALAR TYPE SPECTRAL OPERATORS, INFINITE DIFFERENTIABLE AND GEVREY ULTRADIFFERENTIABLE $C_{0}$-SEMIGROUPS
}

\author{
MARAT V. MARKIN
}

Received 6 November 2003

\begin{abstract}
Necessary and sufficient conditions for a scalar type spectral operator in a Banach space to be a generator of an infinite differentiable or a Gevrey ultradifferentiable $C_{0}$-semigroup are found, the latter formulated exclusively in terms of the operator's spectrum.
\end{abstract}

2000 Mathematics Subject Classification: 47B40, 47D03, 47B15, 34G10.

1. Introduction. Despite what was said in the final remarks to [22], the author did decide to tackle the problems of the generation of infinite differentiable and Gevrey ultradifferentible $C_{0}$-semigroups by a scalar type spectral operator in a complex Banach space. The more so as, in the former case, the task turned out to be more of a challenge than it seemed initially, the existence of a general characterization of infinite differentiable $C_{0}$-semigroups [25] (see also [6, 26]) notwithstanding. In the latter case, such characterizations are not to be found in the plethora of the literature on the subject including such authoritative and exhaustive sources as [6, 9, 11, 15, 26, 28, 31].

In [22], the criteria of a scalar type spectral operator in a complex Banach space being a generator of a $C_{0}$-semigroup and an analytic $C_{0}$-semigroup were found. In the present paper, necessary and sufficient conditions for a scalar type spectral operator in a complex Banach space to be a generator of an infinite differentiable or a Gevrey ultradifferentiable $C_{0}$-semigroup are established. The main purpose is to show that such criteria, as well as those of [22], can be formulated exclusively in terms of the operator's spectrum, without any restrictions on its resolvent behavior. This fact distinguishes the case of scalar type spectral operators and makes the aformentioned results significantly more transparent and purely qualitative.

\section{Preliminaries}

2.1. Scalar type spectral operators. Henceforth, unless otherwise specified, $A$ is a scalar type spectral operator in a complex Banach space $X$ with norm $\|\cdot\|$ and $E_{A}(\cdot)$ is its spectral measure (the resolution of the density), the operator's spectrum $\sigma(A)$ being the support for the latter $[2,5]$.

Note that, in a Hilbert space, the scalar type spectral operators are those similar to the normal ones [29].

For such operators, there has been developed an operational calculus for Borel measurable functions on $\mathbb{C}($ on $\sigma(A))[2,5], F(\cdot)$ being such a function, a new scalar type 
spectral operator

$$
F(A)=\int_{\mathbb{C}} F(\lambda) d E_{A}(\lambda)=\int_{\sigma(A)} F(\lambda) d E_{A}(\lambda)
$$

is defined as follows:

$$
\begin{aligned}
F(A) f: & =\lim _{n \rightarrow \infty} F_{n}(A) f, \quad f \in D(F(A)), \\
D(F(A)) & :=\left\{f \in X \mid \lim _{n \rightarrow \infty} F_{n}(A) f \text { exists }\right\}
\end{aligned}
$$

$(D(\cdot)$ is the domain of an operator), where

$$
F_{n}(\cdot):=F(\cdot) \chi_{\{\lambda \in \sigma(A)|| F(\lambda) \mid \leq n\}}(\cdot), \quad n=1,2, \ldots,
$$

$\left(\chi_{\alpha}(\cdot)\right.$ is the characteristic function of a set $\left.\alpha\right)$, and

$$
F_{n}(A):=\int_{\sigma(A)} F_{n}(\lambda) d E_{A}(\lambda), \quad n=1,2, \ldots,
$$

being the integrals of bounded Borel measurable functions on $\sigma(A)$, are bounded scalar type spectral operators on $X$ defined in the same manner as for normal operators (see, e.g., [4, 27]).

The properties of the spectral measure, $E_{A}(\cdot)$, and the operational calculus underlying the entire subsequent argument are exhaustively delineated in [2, 5]. We just observe here that, due to its strong countable additivity, the spectral measure $E_{A}(\cdot)$ is bounded, that is, there is an $M>0$ such that, for any Borel set $\delta$,

$$
\left\|E_{A}(\delta)\right\| \leq M
$$

see [3].

Observe that, in (2.5), the notation $\|\cdot\|$ was used to designate the norm in the space of bounded linear operators on $X$. We will adhere to this rather common economy of symbols in what follows, adopting the same notation for the norm in the dual space $X^{*}$ as well.

Due to (2.5), for any $f \in X$ and $g^{*} \in X^{*}$ ( $X^{*}$ is the dual space), the total variation $v\left(f, g^{*}, \cdot\right)$ of the complex-valued measure $\left\langle E_{A}(\cdot) f, g^{*}\right\rangle(\langle\cdot, \cdot\rangle$ is the pairing between the space $X$ and its dual, $\left.X^{*}\right)$ is bounded. Indeed,

$$
\begin{aligned}
v(f & \left., g^{*}, \sigma(A)\right) \quad(\delta \text { being an arbitrary Borel subset of } \sigma(A), \text { [3] }) \\
& \leq 4 \sup _{\delta \subseteq \sigma(A)}\left|\left\langle E_{A}(\delta) f, g^{*}\right\rangle\right| \leq 4 \sup _{\delta \subseteq \sigma(A)}\left\|E_{A}(\delta)\right\|\|f\|\left\|g^{*}\right\| \quad(\text { by (2.5)) } \\
& \leq 4 M\|f\|\left\|g^{*}\right\| .
\end{aligned}
$$

For the reader's convenience, we reformulate here [23, Proposition 3.1], heavily relied upon in what follows, which allows to characterize the domains of the Borel measurable functions of a scalar type spectral operator in terms of positive measures (see [23] for a complete proof). 
Proposition 2.1 [23, Proposition 3.1]. Let A be a scalar type spectral operator in a complex Banach space $X$ and $F(\cdot)$ a complex-valued Borel measurable function on $\mathbb{C}$ (on $\sigma(A)$ ). Then $f \in D(F(A))$ if and only if

(i) for any $g^{*} \in X^{*}$,

$$
\int_{\sigma(A)}|F(\lambda)| d v\left(f, g^{*}, \lambda\right)<\infty
$$

(ii) $\sup _{\left\{g^{*} \in X^{*} \mid\left\|g^{*}\right\|=1\right\}} \int_{\{\lambda \in \sigma(A) \| F(\lambda) \mid>n\}}\|F(\lambda)\| d v\left(f, g^{*}, \lambda\right) \rightarrow 0$ as $n \rightarrow \infty$.

Observe that, $F(\cdot)$ being an arbitrary Borel measurable function on $\mathbb{C}$ (on $\sigma(A)$ ), for any $f \in D(F(A)), g^{*} \in X^{*}$, and arbitrary Borel sets $\delta \subseteq \sigma$,

$$
\begin{aligned}
\int_{\sigma} \mid & F(\lambda) \mid d v\left(f, g^{*}, \lambda\right) \quad(\text { see [3] }) \\
& \leq 4 \sup _{\delta \subseteq \sigma}\left|\int_{\delta} F(\lambda) d\left\langle E_{A}(\lambda) f, g^{*}\right\rangle\right| \\
& \left.=4 \sup _{\delta \subseteq \sigma}\left|\int_{\sigma} x_{\delta}(\lambda) F(\lambda) d\left\langle E_{A}(\lambda) f, g^{*}\right\rangle\right| \quad \text { (by the properties of the } \text { o.c. }\right) \\
& \left.=4 \sup _{\delta \subseteq \sigma}\left|\left\langle\int_{\sigma} \chi_{\delta}(\lambda) F(\lambda) d E_{A}(\lambda) f, g^{*}\right\rangle\right| \quad \text { (by the properties of the } \text { o.c. }\right) \\
& =4 \sup _{\delta \subseteq \sigma}\left|\left\langle E_{A}(\delta) E_{A}(\sigma) F(A) f, g^{*}\right\rangle\right| \\
& \leq 4 \sup _{\delta \subseteq \sigma}\left\|E_{A}(\delta) E_{A}(\sigma) F(A) f\right\|\left\|g^{*}\right\| \\
& \leq 4 \sup _{\delta \subseteq \sigma}\left\|E_{A}(\delta)\right\|\left\|E_{A}(\sigma) F(A) f\right\|\left\|g^{*}\right\| \quad(\text { by }(2.5)) \\
& \leq 4 M\left\|E_{A}(\sigma) F(A) f\right\|\left\|g^{*}\right\| \\
& \leq 4 M\left\|E_{A}(\sigma)\right\|\|F(A) f\|\left\|g^{*}\right\| .
\end{aligned}
$$

In particular,

$$
\begin{aligned}
\int_{\sigma(A)} & |F(\lambda)| d v\left(f, g^{*}, \lambda\right) \quad(\text { by }(2.8)) \\
\leq & 4 M\left\|E_{A}(\sigma(A))\right\|\|F(A) f\|\left\|g^{*}\right\| \\
& \left(\text { since } E_{A}(\sigma(A))=I(I \text { is the identity operator on } X)\right) \\
\leq & 4 M\|F(A) f\|\left\|g^{*}\right\| .
\end{aligned}
$$

On account of compactness, the terms spectral measure and operational calculus for scalar type spectral operators, frequently referred to, will be abbreviated to s.m. and o.c., respectively.

Observe also that, as follows directly from the results of [1, 23], if a scalar type spectral operator $A$ generates a $C_{0}$-semigroup $\{T(t) \mid t \geq 0\}$, the latter is of the form

$$
T(t)=e^{t A}, \quad 0 \leq t<\infty .
$$


2.2. The Gevrey classes of vectors. Let $A$ be a linear operator in a Banach space $X$ with norm $\|\cdot\|$,

$$
C^{\infty}(A) \stackrel{\text { def }}{=} \bigcap_{n=0}^{\infty} D\left(A^{n}\right),
$$

and $0 \leq \beta<\infty$.

The sets of vectors

$$
\begin{aligned}
& \mathscr{E}^{\{\beta\}}(A) \stackrel{\text { def }}{=}\left\{f \in C^{\infty}(A) \mid \exists \alpha>0, \exists c>0:\left\|A^{n} f\right\| \leq c \alpha^{n}[n !]^{\beta}, n=0,1, \ldots\right\}, \\
& \mathscr{E}^{(\beta)}(A) \stackrel{\text { def }}{=}\left\{f \in C^{\infty}(A) \mid \forall \alpha>0 \exists c>0:\left\|A^{n} f\right\| \leq c \alpha^{n}[n !]^{\beta}, n=0,1, \ldots\right\}
\end{aligned}
$$

are called the $\beta$ th-order Gevrey classes of the operator A of Roumie's and Beurling's types, respectively.

For $0 \leq \beta<\beta^{\prime}<\infty$,

$$
\mathscr{E}^{(\beta)}(A) \subseteq \mathscr{E}^{\{\beta\}}(A) \subseteq \mathscr{E}^{\left(\beta^{\prime}\right)}(A) \subseteq \mathscr{E}^{\left\{\beta^{\prime}\right\}}(A) \subseteq C^{\infty}(A) .
$$

In particular, $\mathscr{E}^{\{1\}}(A)$ and $\mathscr{E}^{(1)}(A)$ are the well-known classes of analytic and entire vectors, correspondingly $[10,24]$.

Observe that, in the definitions of the Gevrey classes, due to Stirling's formula, one can replace $[n !]^{\beta}$ by $n^{\beta n}$.

According to [17], for a scalar type spectral operator $A$ in a complex Banach space $X$ and $0<\beta<\infty$,

$$
\begin{aligned}
& \mathscr{E}^{\{\beta\}}(A) \supseteq \bigcup_{t>0} D\left(e^{t|A|^{1 / \beta}}\right), \\
& \mathscr{E}^{(\beta)}(A) \supseteq \bigcap_{t>0} D\left(e^{t|A|^{1 / \beta}}\right),
\end{aligned}
$$

the inclusions becoming equalities provided that the space $X$ is reflexive.

2.3. Gevrey ultradifferentiability. A smoothness higher than infinite differentiability ranging up to real analyticity and entireness was introduced for numerical functions by Gevrey in 1918 [7] and is naturally extrapolated to functions with values in a Banach space.

Let $I$ be an interval of the real axis, $\mathbb{R}, C^{\infty}(I, X)$ the set of all $X$-valued functions strongly infinite differentiable on $I$, and $0 \leq \beta<\infty$.

The sets of vectors

$$
\begin{gathered}
\mathscr{E}^{\{\beta\}}(I, X) \stackrel{\text { def }}{=}\left\{g(\cdot) \in C^{\infty}(I, X) \mid \forall[a, b] \subseteq I \exists \alpha>0, \exists c>0:\right. \\
\left.\max _{a \leq t \leq b}\left\|g^{(n)}(t)\right\| \leq c \alpha^{n}[n !]^{\beta}, n=0,1, \ldots\right\}, \\
\mathscr{E}^{(\beta)}(I, X) \stackrel{\text { def }}{=}\left\{g(\cdot) \in C^{\infty}(I, X) \mid \forall[a, b] \subseteq I, \forall \alpha>0 \exists c>0:\right. \\
\left.\max _{a \leq t \leq b}\left\|g^{(n)}(t)\right\| \leq c \alpha^{n}[n !]^{\beta}, n=0,1, \ldots\right\}
\end{gathered}
$$


are the $\beta$ th-order Gevrey classes of strongly ultradifferentiable functions of Roumie's and Beurling's types, respectively (see, e.g., [7, 12, 13, 14]).

Just as above, due to Stirling's formula, one can replace $[n !]^{\beta}$ by $n^{\beta n}$.

For $0 \leq \beta<\beta^{\prime}<\infty$, the following inclusions hold:

$$
\mathscr{E}^{(\beta)}(I, X) \subseteq \mathscr{E}^{\{\beta\}}(I, X) \subseteq \mathscr{E}^{\left(\beta^{\prime}\right)}(I, X) \subseteq \mathscr{E}^{\left\{\beta^{\prime}\right\}}(I, X)
$$

In particular, $\mathscr{E}^{\{1\}}(I, X)$ is the class of all real analytic on $I$ vector functions (i.e., analytically continuable into complex neighborhoods of the interval $I$ ) and $\mathscr{E}^{(1)}(I, X)$ is the class of all entire vector functions (i.e., allowing entire continuations) (for numerical functions, see [16]).

Note that it is well known that the Gevrey classes of functions of orders greater than one are quasianalytic.

3. On the strong smoothness of an orbit of a $C_{0}$-semigroup generated by a scalar type spectral operator. Let $A$ be a scalar type spectral operator generating a $C_{0}$-semigroup $\{T(t) \mid t \geq 0\}$.

Proposition 3.1. Let I be a subinterval of $[0, \infty)$ and $0<\beta<\infty$. Then the restriction of an orbit $T(\cdot) f, f \in X$, to $I$

(i) belongs to $C^{\infty}(I, X)$ if and only if $T(t) f \in C^{\infty}(A)$, for any $t \in I$,

(ii) belongs to $\mathscr{E}^{\{\beta\}}(I, X)$ (resp., $\mathscr{E}(\beta)(I, X)$ ) if and only if $T(t) f \in \mathscr{E}^{\{\beta\}}(A)$ (resp., $\left.\mathscr{E}^{(\beta)}(A)\right)$, for any $t \in I$.

\section{PROOF}

(i) “ONLY IF" PART. Assume that the restriction of an orbit $T(\cdot) f$ of the $C_{0}$-semigroup generated by $A$ to a subinterval $I$ of $[0, \infty)$ belongs to $C^{\infty}(I, X)$.

Taking into account that $T(\cdot) f$ is a weak solution of the evolution equation

$$
y^{\prime}(t)=A y(t)
$$

on $[0, \infty)[1]$, we have, for any $g \in D\left(A^{*}\right)$,

$$
\left\langle T^{\prime}(t) f, g\right\rangle=\frac{d}{d t}\langle T(t) f, g\rangle=\left\langle T(t) f, A^{*} g\right\rangle, \quad t \in I .
$$

Whence, by the closedness of the operator $A$,

$$
T(t) f \in D(A), \quad T^{\prime}(t) f=A T(t) f, \quad \text { for any } t \in I,
$$

(see $[1,8]$ for details).

Let $n>1$. Then, differentiating (3.3) for an arbitrary fixed $t \in I$, we obtain

$$
T^{\prime \prime}(t) f=\lim _{\Delta t \rightarrow 0} \frac{T^{\prime}(t+\Delta t) f-T^{\prime}(t) f}{\Delta t}=\lim _{\Delta t \rightarrow 0} A \frac{T(t+\Delta t) f-T(t) f}{\Delta t},
$$

where the increments $\Delta t$ are such that $t+\Delta \in I$. 
Since

$$
\lim _{\Delta t \rightarrow 0} \frac{T(t+\Delta t) f-T(t) f}{\Delta t}=T^{\prime}(t) f,
$$

by the closedness of $A$, we infer that

$$
T^{\prime}(t) f \in D(A), \quad T^{\prime \prime}(t) f=A T^{\prime}(t) f, \quad \text { for any } t \in I .
$$

Thus, (3.3) and (3.6) imply

$$
T(t) f \in D\left(A^{2}\right), \quad T^{\prime \prime}(t) f=A^{2} T(t) f, \quad \text { for any } t \in I .
$$

Continuing inductively in this manner, we infer that for any $n=1,2, \ldots$,

$$
T(t) f \in C^{\infty}(A), \quad T^{(n)}(t) f=A^{n} T(t) f, \quad t \in I .
$$

"IF" PART. Let $T(\cdot) f$ be an orbit of the $C_{0}$-semigroup generated by $A$ such that

$$
T(t) f \in C^{\infty}(A) \text { for any } t \in I \text {, }
$$

where $I$ is a subinterval of $[0, \infty)$.

Recall that, as was noted in Section 2, the $C_{0}$-semigroup $\{T(t) \mid t \geq 0\}$ generated by $A$ is of the form (2.10).

The fact that

$$
e^{t A} f \in C^{\infty}(A), \quad t \in I,
$$

by [23, Proposition 3.1], implies that, for any $g^{*} \in X^{*}$,

$$
\int_{\sigma(A)}|\lambda|^{n} e^{t \operatorname{Re} \lambda} d v\left(f, g^{*}, \lambda\right)<\infty, \quad n=1,2, \ldots, t \in I .
$$

Given a natural $n$ and an arbitrary fixed $t \in[0, T)$, we choose a segment $[a, b] \subset[0, T)$ $(a<b)$ so that $t$ is its midpoint if $0<t<T$, or $a=0$ if $t=0$. For increments $\Delta t$ such that $a \leq t+\Delta t \leq b$ and any $g^{*} \in X^{*}$, we have

$$
\begin{gathered}
\left|\left\langle\frac{A^{n-1} e^{t+\Delta t} f-A^{n-1} e^{t} f}{\Delta t}-A^{n} e^{t} f, g^{*}\right\rangle\right| \quad(\text { by (2.10) and the properties of the o.c.) } \\
=\left|\left\langle\int_{\sigma(A)}\left[\frac{\lambda^{n-1} e^{(t+\Delta t) \lambda}-\lambda^{n-1} e^{t \lambda}}{\Delta t}-\lambda^{n} e^{t \lambda}\right] d E_{A}(\lambda) f, g^{*}\right\rangle\right|
\end{gathered}
$$

(by the properties of the o.c.)

$$
\begin{aligned}
& \leq\left|\int_{\sigma(A)}\left[\frac{\lambda^{n-1} e^{(t+\Delta t) \lambda}-\lambda^{n-1} e^{t \lambda}}{\Delta t}-\lambda^{n} e^{t \lambda}\right] d\left\langle E_{A}(\lambda) f, g^{*}\right\rangle\right| \\
& \leq \int_{\sigma(A)}\left|\frac{\lambda^{n-1} e^{(t+\Delta t) \lambda}-\lambda^{n-1} e^{t \lambda}}{\Delta t}-\lambda^{n} e^{t \lambda}\right| d v\left(f, g^{*}, \lambda\right)
\end{aligned}
$$

(by the Lebesgue dominated convergence theorem)

$\longrightarrow 0$ as $\Delta t \longrightarrow 0$. 
Indeed, for any $\lambda \in \sigma(A)$,

$$
\begin{aligned}
& \mid \begin{array}{l}
\left|\frac{\lambda^{n-1} e^{(t+\Delta t) \lambda}-\lambda^{n-1} e^{t \lambda}}{\Delta t}-\lambda^{k} e^{t \lambda}\right| \\
\quad \leq\left|\frac{\lambda^{n-1} e^{(t+\Delta t) \lambda}-\lambda^{n-1} e^{t \lambda}}{\Delta t}\right|+\left|\lambda^{n} e^{t \lambda}\right| \quad \text { (by the total change theorem) } \\
\quad \leq \max _{a \leq s \leq b}\left|\lambda^{n} e^{s \lambda}\right|+\left|\lambda^{n} e^{t \lambda}\right| \leq 2|\lambda|^{n} \max _{a \leq s \leq b} e^{s \operatorname{Re} \lambda}
\end{array} \\
& \quad \leq 2\left\{\begin{array}{l}
|\lambda|^{n} e^{a \operatorname{Re} \lambda}, \quad \text { if } \operatorname{Re} \lambda<0, \quad(\text { by }(3.11), \text { considering that } a, b \in I) \\
|\lambda|^{n} e^{b \operatorname{Re} \lambda}, \quad \text { if } \operatorname{Re} \lambda \geq 0,
\end{array}\right. \\
& \quad \in \mathscr{L}^{1}\left(\sigma(A), v\left(f, g^{*}, \cdot\right)\right), \quad n=1,2, \ldots, \\
& \left|\frac{\lambda^{n-1} e^{(t+\Delta t) \lambda}-\lambda^{n-1} e^{t \lambda}}{\Delta t}-\lambda^{n} e^{t \lambda}\right| \rightarrow 0 \quad \text { as } \Delta t \rightarrow 0 .
\end{aligned}
$$

We have shown that, for any $t \in I$ and an arbitrary $n=1,2, \ldots$,

$$
\frac{A^{n-1} e^{t+\Delta t} f-A^{n-1} e^{t} f}{\Delta t} \stackrel{w}{\longrightarrow} A^{n} y(t) \longrightarrow 0 \quad \text { as } \Delta t \rightarrow 0 .
$$

Thus, we have proved that, for any $g^{*} \in X^{*}$,

$$
\frac{d^{n}}{d t^{n}}\left\langle e^{t A} f, g^{*}\right\rangle=\left\langle A^{n} e^{t A} f, g^{*}\right\rangle, \quad n=1,2, \ldots, t \in I .
$$

Now, let

$$
\Delta_{n}:=\{\lambda \in \mathbb{C}|| \lambda \mid \leq n\} .
$$

We fix an arbitrary natural $k$ and consider the sequence of functions

$$
E_{A}\left(\Delta_{n}\right) A^{k} e^{t A} f, \quad n=1,2, \ldots, t \in I .
$$

By the properties of the o.c.,

$$
\begin{aligned}
& E_{A}\left(\Delta_{n}\right) A^{k} e^{t A} \\
& \left.=\int_{\mathbb{C}} \chi_{\Delta_{n}}(\lambda) \lambda^{k} e^{t \lambda} d E_{A}(\lambda) \quad \text { (where } \chi_{\Delta_{n}}(\cdot) \text { is the characteristic function of the set } \Delta_{n}\right) \\
& =\int_{\mathbb{C}}\left[\lambda \chi_{\Delta_{n}}(\lambda)\right]^{k} e^{t \lambda x_{\Delta_{n}}(\lambda)} d E_{A}(\lambda) \\
& =\left[A E_{A}\left(\Delta_{n}\right)\right]^{k} e^{t A E_{A}\left(\Delta_{n}\right)} .
\end{aligned}
$$

Since, by the properties of the o.c., for any natural $n$, the operator $A E_{A}\left(\Delta_{n}\right)$ is a bounded operator on $X\left(\left\|A E_{A}\left(\Delta_{n}\right)\right\| \leq 4 M n\right)$ [5], the vector function

$$
E_{A}\left(\Delta_{n}\right) A^{k} e^{t A} f=\left[A E_{A}\left(\Delta_{n}\right)\right]^{k} e^{t A E_{A}\left(\Delta_{n}\right)} f, \quad n=1,2, \ldots, t \in I,
$$

is strongly continuous. 
For an arbitrary segment $[a, b] \subseteq I$, we have

$$
\begin{aligned}
\sup _{a \leq t \leq b} & \left\|A^{k} e^{t A} f-E_{A}\left(\Delta_{n}\right) A^{k} e^{t A} f\right\| \quad \text { (by the properties of the o.c.) } \\
= & \sup _{a \leq t \leq b}\left\|\int_{\{\lambda \in \sigma(A)|| \lambda \mid>n\}} \lambda^{k} e^{t \lambda} d E_{A}(\lambda) f\right\|
\end{aligned}
$$

(as follows from the Hahn-Banach theorem)

$$
=\sup _{a \leq t \leq b} \sup _{\left\{g^{*} \in X^{*} \mid\left\|g^{*}\right\|=1\right\}}\left|\left\langle\int_{\{\lambda \in \sigma(A)|| \lambda \mid>n\}} \lambda^{k} e^{t \lambda} d E_{A}(\lambda) f, g^{*}\right\rangle\right|
$$

(by the properties of the o.c.)

$$
\begin{aligned}
& =\sup _{a \leq t \leq b} \sup _{\left\{g^{*} \in X^{*} \mid\left\|g^{*}\right\|=1\right\}}\left|\int_{\{\lambda \in \sigma(A)|| \lambda \mid>n\}} \lambda^{k} e^{t \lambda} d\left\langle E_{A}(\lambda) f, g^{*}\right\rangle\right| \\
& \leq \sup _{a \leq t \leq b} \sup _{\left\{g^{*} \in X^{*} \mid\left\|g^{*}\right\|=1\right\}} \int_{\{\lambda \in \sigma(A)|| \lambda \mid>n\}}|\lambda|^{k} e^{t \operatorname{Re} \lambda} d v\left(f, g^{*}, \lambda\right) \\
& \leq \sup _{\left\{g^{*} \in X^{*} \mid\left\|g^{*}\right\|=1\right\}} \sup _{a \leq t \leq b}\left[\int_{\{\lambda \in \sigma(A)|| \lambda \mid>n, \operatorname{Re} \lambda \leq 0\}}|\lambda|^{k} e^{t \operatorname{Re} \lambda} d v\left(f, g^{*}, \lambda\right)\right. \\
& \left.+\int_{\{\lambda \in \sigma(A)|| \lambda \mid>n, \operatorname{Re} \lambda>0\}}|\lambda|^{k} e^{t \operatorname{Re} \lambda} d v\left(f, g^{*}, \lambda\right)\right] \\
& \leq \sup _{\left\{g^{*} \in X^{*} \mid\left\|g^{*}\right\|=1\right\}} \int_{\{\lambda \in \sigma(A)|| \lambda \mid>n, \operatorname{Re} \lambda \leq 0\}}|\lambda|^{k} e^{a \operatorname{Re} \lambda} d v\left(f, g^{*}, \lambda\right)
\end{aligned}
$$$$
+\sup _{\left\{g^{*} \in X^{*} \mid\left\|g^{*}\right\|=1\right\}} \int_{\{\lambda \in \sigma(A)|| \lambda \mid>n, \operatorname{Re} \lambda>0\}}|\lambda|^{k} e^{b \operatorname{Re} \lambda} d v\left(f, g^{*}, \lambda\right)
$$$$
\leq \sup _{\left\{g^{*} \in X^{*} \mid\left\|g^{*}\right\|=1\right\}} \int_{\{\lambda \in \sigma(A)|| \lambda \mid>n\}}|\lambda|^{k} e^{a \operatorname{Re} \lambda} d v\left(f, g^{*}, \lambda\right)
$$$$
+\sup _{\left\{g^{*} \in X^{*} \mid\left\|g^{*}\right\|=1\right\}} \int_{\{\lambda \in \sigma(A)|| \lambda \mid>n\}}|\lambda|^{k} e^{b \operatorname{Re} \lambda} d v\left(f, g^{*}, \lambda\right) \quad \text { (by (2.8)) }
$$

$$
\begin{aligned}
& \leq 4 M\left[\sup _{\left\{g^{*} \in X^{*} \mid\left\|g^{*}\right\|=1\right\}}\left\|E_{A}(\{\lambda \in \sigma(A)|| \lambda \mid>n\}) A^{k} e^{a A} f\right\|\left\|g^{*}\right\|\right. \\
& \left.\quad+\sup _{\left\{g^{*} \in X^{*} \mid\left\|g^{*}\right\|=1\right\}}\left\|E_{A}(\{\lambda \in \sigma(A)|| \lambda \mid>n\}) A^{k} e^{b A} f\right\|\left\|g^{*}\right\|\right] \\
& \leq 4 M\left[\left\|E_{A}(\{\lambda \in \sigma(A)|| \lambda \mid>n\}) A^{k} e^{a A} f\right\|+\left\|E_{A}(\{\lambda \in \sigma(A)|| \lambda \mid>n\}) A^{k} e^{b A} f\right\|\right]
\end{aligned}
$$
(by the strong continuity of the s.m.)

$\longrightarrow 0$ as $n \longrightarrow \infty$.

Therefore, the vector function

$$
A^{k} e^{t A} f, \quad k=1,2, \ldots, t \in I,
$$

is strongly continuous, being the uniform limit of a sequence of strongly continuous functions on an arbitrary segment $[a, b] \subseteq I$. 
We fix an arbitrary $a \in I$ and integrate (3.15) for $n=1$ between $a$ and an arbitrary $t \in I$. Considering the strong continuity of $A e^{t A} f, t \in I$, we have

$$
\left\langle e^{t A} f-e^{a A} f, g^{*}\right\rangle=\left\langle\int_{a}^{t} A e^{s A} f d s, g^{*}\right\rangle, \quad g^{*} \in X^{*} .
$$

Whence, as follows from the Hahn-Banach theorem,

$$
e^{t A} f-e^{a A} f=\int_{a}^{t} A e^{s A} f d s
$$

By the strong continuity of $A e^{t A} f, t \in I$, we infer that

$$
\frac{d}{d t} e^{t A} f=A e^{t A} f, \quad t \in I
$$

Consequently, by (3.15) for $n=2$,

$$
\frac{d}{d t}\left\langle\frac{d}{d t} e^{t A} f, g^{*}\right\rangle=\left\langle A^{2} e^{t A} f, g^{*}\right\rangle, \quad t \in I
$$

Whence, analogously,

$$
\frac{d^{2}}{d t^{2}} e^{t A} f=A^{2} e^{t A} f, \quad t \in I
$$

Continuing inductively in this manner, we infer that, for any natural $n$,

$$
\frac{d^{n}}{d t^{n}} e^{t A} f=A^{n} e^{t A} f, \quad t \in I
$$

(ii) “ONLY IF" PART. Assume that an orbit $T(\cdot) f, f \in X$, of the $C_{0}$-semigroup $\{T(t) \mid$ $t \geq 0\}$ generated by $A$ restricted to a subinterval $I \subseteq[0, \infty)$ belongs to $\mathscr{E}^{\{\beta\}}(I, X)$ (resp., $\left.\mathscr{E}^{(\beta)}(I, X)\right)$.

This necessarily implies that $T(\cdot) f \in C^{\infty}(I, H)$. Whence, by (i),

$$
T(t) f \in C^{\infty}(A), \quad T^{(n)}(t) f=A^{n} T(t) f, \quad n=1,2, \ldots, t \in I .
$$

Furthermore, the fact that the restriction of $y(\cdot)$ to $I$ belongs to the class $\mathscr{E}^{\{\beta\}}(I, X)$ (resp., $\left.\mathscr{E}^{(\beta)}(I, X)\right)$ implies that, for an arbitrary $t \in I$, a certain (any) $\alpha>0$, and a certain $c>0$,

$$
\left\|A^{n} T(t) f\right\|=\left\|T^{(n)}(t) f\right\| \leq c \alpha^{n}[n !]^{\beta}, \quad n=0,1, \ldots
$$

Therefore,

$$
T(t) f \in \mathscr{E}^{\{\beta\}}(A)\left(\text { resp., } \mathscr{E}^{(\beta)}(A)\right), \quad t \in I .
$$


"IF" PART. Let an orbit $T(\cdot) f, f \in X$, of the $C_{0}$-semigroup $\{T(t) \mid t \geq 0\}$ generated by $A$ be such that (3.30) holds, where $I$ is a subinterval of $[0, \infty)$.

Hence, for arbitrary $t \in I$ and some (any) $\alpha(t)>0$, there is such a $c(t, \alpha)>0$ that

$$
\left\|A^{n} T(t) f\right\| \leq c(t, \alpha) \alpha(t)^{n}[n !]^{\beta}, \quad n=0,1,2, \ldots
$$

The inclusions

$$
\mathscr{E}^{(\beta)}(A) \subseteq \mathscr{E}^{\{\beta\}}(A) \subseteq C^{\infty}(A)
$$

imply, by (i), that (3.28) holds. Recall that

$$
T(t) f=e^{t A} f, \quad 0 \leq t<\infty .
$$

We fix an arbitrary subsegment $[a, b] \subseteq I$. For $n=0,1, \ldots$, we have

$$
\begin{aligned}
& \max _{a \leq t \leq b}\left\|T^{(n)}(t) f\right\| \\
& =\max _{a \leq t \leq b}\left\|A^{n} T(t) f\right\| \\
& =\max _{a \leq t \leq b}\left\|A^{n} e^{t A} f\right\| \quad \text { (by the properties of the o.c. and the Hahn-Banach theorem) } \\
& =\max _{a \leq t \leq b} \sup _{\left\{g^{*} \in X^{*}\left\|g^{*}\right\|=1\right\}}\left|\left\langle\int_{\sigma(A)} \lambda^{n} e^{t \lambda} d E_{A}(\lambda) f, g^{*}\right\rangle\right| \quad \text { (by the properties of the } \text { o.c.) } \\
& \leq \max _{a \leq t \leq b} \sup _{\left\{g^{*} \in X^{*} \mid\left\|g^{*}\right\|=1\right\}}\left|\int_{\sigma(A)} \lambda^{n} e^{t \lambda} d\left\langle E_{A}(\lambda) f, g^{*}\right\rangle\right| \\
& \leq \max _{a \leq t \leq b} \sup _{\left\{g^{*} \in X^{*} \mid\left\|g^{*}\right\|=1\right\}} \int_{\sigma(A)}|\lambda|^{n} e^{t \operatorname{Re} \lambda} d v\left(f, g^{*}, \lambda\right) \\
& =\sup _{\left\{g^{*} \in X^{*} \mid\left\|g^{*}\right\|=1\right\}} \max _{a \leq t \leq b}\left[\int_{\{\lambda \in \sigma(A) \mid \operatorname{Re} \lambda \leq 0\}}|\lambda|^{n} e^{t \operatorname{Re} \lambda} d v\left(f, g^{*}, \lambda\right)\right. \\
& \left.+\int_{\{\lambda \in \sigma(A) \mid \operatorname{Re} \lambda>0\}}|\lambda|^{n} e^{t \operatorname{Re} \lambda} d v\left(f, g^{*}, \lambda\right)\right] \\
& \leq \sup _{\left\{g^{*} \in X^{*} \mid\left\|g^{*}\right\|=1\right\}} \int_{\{\lambda \in \sigma(A) \mid \operatorname{Re} \lambda \leq 0\}}|\lambda|^{n} e^{a \operatorname{Re} \lambda} d v\left(f, g^{*}, \lambda\right) \\
& +\sup _{\left\{g^{*} \in X^{*} \mid\left\|g^{*}\right\|=1\right\}} \int_{\{\lambda \in \sigma(A) \mid \operatorname{Re} \lambda>0\}}|\lambda|^{n} e^{b \operatorname{Re} \lambda} d v\left(f, g^{*}, \lambda\right) \\
& \leq \sup _{\left\{g^{*} \in X^{*} \mid\left\|g^{*}\right\|=1\right\}} \int_{\sigma(A)}|\lambda|^{n} e^{a \operatorname{Re} \lambda} d v\left(f, g^{*}, \lambda\right) \\
& +\sup _{\left\{g^{*} \in X^{*} \mid\left\|g^{*}\right\|=1\right\}} \int_{\sigma(A)}|\lambda|^{n} e^{b \operatorname{Re} \lambda} d v\left(f, g^{*}, \lambda\right) \quad(\text { by (2.9)) } \\
& \leq \sup _{\left\{g^{*} \in X^{*} \mid\left\|g^{*}\right\|=1\right\}} 4 M\left\|A^{n} e^{a A} f\right\|\left\|g^{*}\right\|+\sup _{\left\{g^{*} \in X^{*} \mid\left\|g^{*}\right\|=1\right\}} 4 M\left\|A^{n} e^{b A} f\right\|\left\|g^{*}\right\| \\
& \leq 4 M\left[\left\|A^{n} e^{a A} f\right\|+\left\|A^{n} e^{b A} f\right\|\right]=4 M\left[\left\|A^{n} y(a)\right\|+\left\|A^{n} y(b)\right\|\right] \quad \text { (by (3.28)) } \\
& =4 M\left[\left\|y^{(n)}(a)\right\|+\left\|y^{(n)}(b)\right\|\right] \text {. }
\end{aligned}
$$


Hence, in view of (3.31), we obtain

$$
\begin{aligned}
\max _{a \leq t \leq b} & \left\|T^{(n)}(t) f\right\| \\
& \leq 4 M[c(a, \alpha)+c(b, \alpha)] \max [\alpha(a), \alpha(b)]^{n}[n !]^{\beta}, \quad n=0,1,2, \ldots,
\end{aligned}
$$

which implies that the restriction of $T(\cdot) f$ to the subinterval $I \subseteq[0, T)$ belongs to the Gevrey class $\mathscr{E}^{\{\beta\}}(I, X)$ (resp., $\left.\mathscr{E}^{(\beta)}(I, X)\right)$.

4. Infinite differentiable $C_{0}$-semigroups. Recall that a $C_{0}$-semigroup $\{T(t) \mid t \geq 0\}$ in a Banach space $X$ is said to be infinite differentiable (a $C^{\infty}$-semigroup) if, for any $f \in X$, the orbit $T(\cdot) f$ is infinite differentiable on $(0, \infty)$ in the strong sense. Note that, due to the semigroup property $T(t+s)=T(t) T(s), t, s \geq 0$, the first-order strong differentiability of an orbit on $(0, \infty)$ immediately implies its infinite strong differentiability on $(0, \infty)$.

THEOREM 4.1. A $C_{0}$-semigroup generated by a scalar type spectral operator $A$ in a complex Banach space $X$ is infinite differentiable if and only if, for an arbitrary positive $b$, there is a real a such that

$$
\operatorname{Re} \lambda \leq a-b \ln |\operatorname{Im} \lambda|, \quad \lambda \in \sigma(A) .
$$

\section{ProOF}

"ONLY IF" PART. This part immediately follows from the general criterion of the generation of infinite differentiable $C_{0}$-semigroups [25] (see also [6, 26]).

"IF" PART. Here, unlike in [22], resorting to the general criterion, that is, proving that there is a $C>0$ such that in the region

$$
R_{b}:=\{\lambda \in \mathbb{C}|\operatorname{Re} \lambda>a-b \ln | \operatorname{Im} \lambda \mid\} \subseteq \rho(A)
$$

$(\rho(\cdot)$ is the resolvent set of an operator) the estimate

$$
\|R(\lambda, A)\| \leq C|\operatorname{Im} \lambda|
$$

holds, brings about rather formidable difficulties. The reader could try evaluating the distance from a point $\lambda \in R_{b}$ to the boundary of the region $R_{b}$ such an approach would inevitably entail.

Utilizing the general criterion not being an option, we are to prove directly that all the orbits of the semigroup generated by $A$ are strongly differentiable on $(0, \infty)$.

Since $A$ generates a $C_{0}$-semigroup, the latter, as was shown in [22], consists of its exponentials,

$$
e^{t A}=\int_{\sigma(A)} e^{t \lambda} d E_{A}(\lambda), \quad t \geq 0
$$


and there is a real $\omega$ such that

$$
\operatorname{Re} \lambda \leq \omega, \quad \lambda \in \sigma(A)
$$

Without loss of generality, we can regard that

$$
\operatorname{Re} \lambda \leq 0, \quad \lambda \in \sigma(A),
$$

that is, we deal with a contraction semigroup. Indeed, otherwise, we can consider the $C_{0}$-semigroup $T(t):=e^{-\omega t} e^{t A}, t \geq 0$, which, evidently, satisfies (4.1).

We need to show that, for any $f \in X$,

$$
e^{t A} f \in D(A), \quad 0<t<\infty .
$$

Let $0<t<\infty$. Since the constant $b$ can acquire arbitrary positive values, we can set $b:=1 / t$. Then, for any Borel set $\sigma \subseteq \sigma(A)$ and arbitrary $f \in X$ and $g^{*} \in X^{*}$,

$$
\begin{aligned}
\int_{\sigma} \mid & \lambda \mid e^{t \operatorname{Re} \lambda} d v\left(f, g^{*}, \lambda\right) \\
\leq & \int_{\sigma}(|\operatorname{Re} \lambda|+|\operatorname{Im} \lambda|) e^{t \operatorname{Re} \lambda} d v\left(f, g^{*}, \lambda\right) \\
& \left(\text { for } \lambda \in \sigma, \operatorname{Re} \lambda \leq \min (0, a-b \ln |\operatorname{Im} \lambda|) \Longrightarrow \operatorname{Re} \lambda \leq 0 \text { and }|\operatorname{Im} \lambda| \leq e^{b^{-1}(a-\operatorname{Re} \lambda)}\right) \\
\leq & \int_{\sigma}\left(-\operatorname{Re} \lambda+e^{a b^{-1}} e^{b^{-1}(-\operatorname{Re} \lambda)}\right) e^{t \operatorname{Re} \lambda} d v\left(f, g^{*}, \lambda\right) \quad\left(\text { since } x \leq e^{x}, 0 \leq x<\infty\right) \\
\leq & \int_{\sigma}\left(b e^{b^{-1}(-\operatorname{Re} \lambda)}+e^{a b^{-1}} e^{b^{-1}(-\operatorname{Re} \lambda)}\right) e^{t \operatorname{Re} \lambda} d v\left(f, g^{*}, \lambda\right) \\
= & {\left[b+e^{a b^{-1}}\right] \int_{\sigma} e^{\left(t-b^{-1}\right) \operatorname{Re} \lambda} d v\left(f, g^{*}, \lambda\right) \quad\left(\text { by the choice } b=\frac{1}{t}\right) } \\
= & {\left[\frac{1}{t}+e^{a t}\right] \int_{\sigma} 1 d v\left(f, g^{*}, \lambda\right)=\left[\frac{1}{t}+e^{a t}\right] v\left(f, g^{*}, \sigma\right) . }
\end{aligned}
$$

This estimate, by [23, Proposition 3.1], implies (4.7).

Indeed,

(i) for any $f \in X$ and $g^{*} \in X^{*}$, we have

$$
\begin{aligned}
\int_{\sigma(A)}|\lambda| e^{t \operatorname{Re} \lambda} d v\left(f, g^{*}, \lambda\right) & \leq\left[\frac{1}{t}+e^{a t}\right] v\left(f, g^{*}, \sigma(A)\right) \quad(\text { by }(2.6)) \\
& \leq 4 M\left[\frac{1}{t}+e^{a t}\right]\|f\|\left\|g^{*}\right\|, \quad 0<t<\infty,
\end{aligned}
$$


(ii) analogously, for any $0<t<\infty$ and an arbitrary $f \in X$,

$$
\begin{aligned}
& \sup _{\left\{g^{*} \in X^{*} \mid\left\|g^{*}\right\|=1\right\}} \int_{\left\{\lambda \in \sigma(A)|| \lambda \mid e^{t \operatorname{Re} \lambda>n\}}\right.}|\lambda| e^{t \operatorname{Re} \lambda} d v\left(f, g^{*}, \lambda\right) \\
& \quad \leq \sup _{\left\{g^{*} \in X^{*} \mid\left\|g^{*}\right\|=1\right\}}\left[\frac{1}{t}+e^{a t}\right] \int_{\left\{\lambda \in \sigma(A) \| \lambda \mid e^{t \operatorname{Re} \lambda>n\}}\right.} 1 d v\left(f, g^{*}, \lambda\right) \quad(\text { by (2.8)) } \\
& \quad \leq\left[\frac{1}{t}+e^{a t}\right] \sup _{\left\{g^{*} \in X^{*} \mid\left\|g^{*}\right\|=1\right\}} 4 M\left\|E_{A}\left(\left\{\lambda \in \sigma(A)|| \lambda \mid e^{t \operatorname{Re} \lambda}>n\right\}\right) f\right\|\left\|g^{*}\right\| \\
& \quad \leq 4 M\left[\frac{1}{t}+e^{a t}\right]\left\|E_{A}\left(\left\{\lambda \in \sigma(A)|| \lambda \mid e^{t \operatorname{Re} \lambda}>n\right\}\right) f\right\|
\end{aligned}
$$

(by the strong continuity of the s.m.)

$\longrightarrow 0$, as $n \longrightarrow \infty$.

Thus, by [23, Proposition 3.1], (4.7) holds.

5. Gevrey ultradifferentiable $C_{0}$-semigroups. Let $0<\beta<\infty$. We will call a $C_{0}$-semigroup $\{T(t) \mid t \geq 0\}$ in a Banach space $X$ an $\mathscr{E}^{\{\beta\}}$-semigroup (resp., an $\mathscr{E}^{(\beta)}$-semigroup) if, for any $f \in X$, the orbit $T(\cdot) f$ belongs to the Gevrey class $\mathscr{E}^{\{\beta\}}((0, \infty), X)$ (resp., $\left.\mathscr{E}^{(\beta)}((0, \infty), X)\right)$. We will call a $C_{0}$-semigroup a Gevrey ultradifferentiable semigroup if, for some $0<\beta<\infty$, it is an $\mathscr{E}^{\{\beta\}}$-semigroup or, which, due to inclusions (2.16), is the same, an $\mathscr{E}^{(\beta)}$-semigroup.

THEOREM 5.1. Let $1 \leq \beta<\infty$. A $C_{0}$-semigroup generated by a scalar type spectral operator $A$ in a complex Banach space $X$ is an $\mathscr{E}^{\{\beta\}}$-semigroup if and only if there are a positive $b$ and a real $a$ such that

$$
\operatorname{Re} \lambda \leq a-b|\operatorname{Im} \lambda|^{1 / \beta}, \quad \lambda \in \sigma(A) .
$$

\section{PROOF}

"IF" PART. As is easily seen, the sufficiency condition of Theorem 5.1 is stronger than that of Theorem 4.1. Therefore, by Theorem 4.1, we infer that $A$ generates an infinitely differentiable $C_{0}$-semigroup consisting, according to [22], of its exponentials presented in (4.4). For any $f \in X$ and $n=1,2, \ldots$,

$$
\frac{d^{n}}{d t^{n}} e^{t A} f=A^{n} e^{t A} f, \quad 0<t<\infty
$$

According to Proposition 3.1, we need to show that, for any $f \in X$,

$$
e^{t A} f \in \mathscr{E}^{\{\beta\}}(A), \quad 0<t<\infty .
$$

In view of inclusions (2.14), it suffices to show that

$$
e^{t A} f \in \bigcup_{s>0} D\left(e^{s|A|^{1 / \beta}}\right), \quad 0<t<\infty .
$$

We fix an arbitrary Borel subset $\sigma$ of $\sigma(A)$ and an arbitrary $t>0$. We also set $s:=$ $t /\left[1+(2 / b)^{\beta}\right]^{1 / \beta}>0$ (such a peculiar choice of $s$ will make sense later). 
For any $f \in X$ and $g^{*} \in X^{*}$,

$$
\begin{aligned}
\int_{\sigma} e^{s|\lambda|^{1 / \beta}} e^{t \operatorname{Re} \lambda} d v\left(f, g^{*}, \lambda\right) \\
=\int_{\{\lambda \in \sigma \mid \operatorname{Re} \lambda \leq \min (-1, a)\}} e^{s|\lambda|^{1 / \beta}} e^{t \operatorname{Re} \lambda} d v\left(f, g^{*}, \lambda\right) \\
\quad+\int_{\{\lambda \in \sigma \mid \min (-1, a)<\operatorname{Re} \lambda \leq a\}} e^{s|\lambda|^{1 / \beta}} e^{t \operatorname{Re} \lambda} d v\left(f, g^{*}, \lambda\right)<\infty .
\end{aligned}
$$

Indeed, the latter integral is finite due to the boundedness of the set $\{\lambda \in \sigma \mid \min (-1, a)$ $<\operatorname{Re} \lambda \leq a\}$ (note that, for $a \leq-1$, the set is, obviously, empty), the continuity of the integrated function, and the finiteness of the positive measure $v\left(f, g^{*}, \cdot\right)$ (see (2.6)).

For the former of the above two integrals, we have

$$
\begin{gathered}
\int_{\{\lambda \in \sigma \mid \operatorname{Re} \lambda \leq \min (-1, a)\}} e^{s|\lambda|^{1 / \beta}} e^{t \operatorname{Re} \lambda} d v\left(f, g^{*}, \lambda\right) \\
\quad\left(\lambda \in \sigma, \operatorname{Re} \lambda \leq \min (-1, a) \Longrightarrow \operatorname{Re} \lambda \leq-1 \text { and }|\operatorname{Im} \lambda| \leq b^{-\beta}(a-\operatorname{Re} \lambda)^{\beta}\right) \\
\leq \int_{\{\lambda \in \sigma \mid \operatorname{Re} \lambda \leq \min (-1, a)\}} e^{s\left[-\operatorname{Re} \lambda+b^{-\beta}(a-\operatorname{Re} \lambda)^{\beta}\right]^{1 / \beta}} e^{t \operatorname{Re} \lambda} d v\left(f, g^{*}, \lambda\right) .
\end{gathered}
$$

We consider separately the two possible cases $a \leq 0$ and $a>0$.

If $a \leq 0$, then $a-\operatorname{Re} \lambda \leq-2 \operatorname{Re} \lambda$ for all $\lambda$ 's such that $\operatorname{Re} \lambda \leq \min (-1, a)$, and we have

$$
\begin{aligned}
& \int_{\{\lambda \in \sigma \mid \operatorname{Re} \lambda \leq \min (-1, a)\}} e^{s|\lambda|^{1 / \beta}} e^{t \operatorname{Re} \lambda} d v\left(f, g^{*}, \lambda\right) \\
& \leq \int_{\{\lambda \in \sigma \mid \operatorname{Re} \lambda \leq \min (-1, a)\}} e^{s\left[-\operatorname{Re} \lambda+b^{-\beta}(-2 \operatorname{Re} \lambda)^{\beta}\right]^{1 / \beta}} e^{t \operatorname{Re} \lambda} d v\left(f, g^{*}, \lambda\right) \\
& \left.\quad \text { (since, for } 1 \leq \beta<\infty, x \leq x^{\beta}, x \geq 1\right) \\
& \leq \int_{\{\lambda \in \sigma \mid \operatorname{Re} \lambda \leq \min (-1, a)\}} e^{s\left[(-\operatorname{Re} \lambda)^{\beta}+(2 / b)^{\beta}(-\operatorname{Re} \lambda)^{\beta}\right]^{1 / \beta}} e^{t \operatorname{Re} \lambda} d v\left(f, g^{*}, \lambda\right) \\
& =\int_{\{\lambda \in \sigma \mid \operatorname{Re} \lambda \leq \min (-1, a)\}} e^{\left(t-s\left[1+(2 / b)^{\beta}\right]^{1 / \beta}\right) \operatorname{Re} \lambda} d v\left(f, g^{*}, \lambda\right) \\
& \quad\left(\text { by the choice } s=\frac{t}{\left[1+(2 / b)^{\beta}\right]^{1 / \beta}}\right) \\
& =\int_{\{\lambda \in \sigma \mid \operatorname{Re} \lambda \leq \min (-1, a)\}} 1 d v\left(f, g^{*}, \lambda\right) \\
& =v\left(f, g^{*},\{\lambda \in \sigma \mid \operatorname{Re} \lambda \leq \min (-1, a)\}\right) \quad(\text { by }(2.6)) \\
& \leq 4 M\|f\|\left\|g^{*}\right\|<\infty .
\end{aligned}
$$

If $a>0$,

$$
\begin{aligned}
& \int_{\{\lambda \in \sigma \mid \operatorname{Re} \lambda \leq \min (-1, a)\}} e^{s|\lambda|^{1 / \beta}} e^{t \operatorname{Re} \lambda} d v\left(f, g^{*}, \lambda\right) \\
& =\int_{\{\lambda \in \sigma \mid \operatorname{Re} \lambda \leq \min (-1,-a)\}} e^{s|\lambda|^{1 / \beta}} e^{t \operatorname{Re} \lambda} d v\left(f, g^{*}, \lambda\right) \\
& \quad+\int_{\{\lambda \in \sigma \mid \min (-1,-a)<\operatorname{Re} \lambda \leq-1\}} e^{s|\lambda|^{1 / \beta}} e^{t \operatorname{Re} \lambda} d v\left(f, g^{*}, \lambda\right)<\infty .
\end{aligned}
$$


Indeed, the latter integral is finite due to the boundedness of the set $\{\lambda \in \sigma \mid$ $\min (-a,-1)<\operatorname{Re} \lambda \leq-1\}$ (note that, for $a \geq 1$, the set is, obviously, empty), the continuity of the integrated function, and the finiteness of the positive measure $v\left(f, g^{*}, \cdot\right)$ (see (2.6)).

The former of the above two integrals is finite as well:

$$
\begin{aligned}
& \left.\int_{\{\lambda \in \sigma \mid \operatorname{Re} \lambda \leq \min (-1,-a)\}} e^{s|\lambda|^{1 / \beta}} e^{t \operatorname{Re} \lambda} d v\left(f, g^{*}, \lambda\right) \quad \text { (since, for } x \leq-a, a-x \leq-2 x\right) \\
& \quad \leq \int_{\{\lambda \in \sigma \mid \operatorname{Re} \lambda \leq \min (-1,-a)\}} e^{s\left[-\operatorname{Re} \lambda+b^{-\beta}(-2 \operatorname{Re} \lambda)^{\beta}\right]^{1 / \beta}} e^{t \operatorname{Re} \lambda} d v\left(f, g^{*}, \lambda\right) \\
& \left.\quad \text { (since, for } 1 \leq \beta<\infty, x \leq x^{\beta}, x \geq 1\right) \\
& \quad \int_{\{\lambda \in \sigma \mid \operatorname{Re} \lambda \leq \min (-1,-a)\}} e^{s\left[(-\operatorname{Re} \lambda)^{\beta}+(2 / b)^{\beta}(-\operatorname{Re} \lambda)^{\beta}\right]^{1 / \beta}} e^{t \operatorname{Re} \lambda} d v\left(f, g^{*}, \lambda\right) \\
& \quad \int_{\{\lambda \in \sigma \mid \operatorname{Re} \lambda \leq \min (-1,-a)\}} e^{\left(t-s\left[1+(2 / b)^{\beta}\right]^{1 / \beta}\right) \operatorname{Re} \lambda} d v\left(f, g^{*}, \lambda\right) \\
& \quad=\int_{\{\lambda \in \sigma \mid \operatorname{Re} \lambda \leq \min (-1,-a)\}} 1 d v\left(f, g^{*}, \lambda\right) \quad\left(\text { by the choice } s=\frac{t}{\left[1+(2 / b)^{\beta}\right]^{1 / \beta}}\right) \\
& =v\left(f, g^{*},\{\lambda \in \sigma \mid \operatorname{Re} \lambda \leq \min (-1,-a)\}\right) \quad(\text { by (2.6)) } \\
& \leq 4 M\|f\|\left\|g^{*}\right\|<\infty .
\end{aligned}
$$

Thus, we have proved that, for an arbitrary Borel subset $\sigma \subseteq \sigma(A)$, any $f \in X$, and $g^{*} \in X^{*}$,

$$
\int_{\sigma} e^{s|\lambda|^{1 / \beta}} e^{t \operatorname{Re} \lambda} d v\left(f, g^{*}, \lambda\right)<\infty, \quad t>0
$$

with $s=t /\left[1+(2 / b)^{\beta}\right]^{1 / \beta}>0$.

This, in particular, implies that, for any $f \in X$ and $g^{*} \in X^{*}$,

$$
\int_{\sigma(A)} e^{s|\lambda|^{1 / \beta}} e^{t \operatorname{Re} \lambda} d v\left(f, g^{*}, \lambda\right)<\infty, \quad t>0
$$

with $s=t /\left[1+(2 / b)^{\beta}\right]^{1 / \beta}>0$.

Furthermore, for any $f \in X, g^{*} \in X^{*}, t>0$, and $s=t /\left[1+(2 / b)^{\beta}\right]^{1 / \beta}>0$,

$$
\sup _{\left\{g^{*} \in X^{*} \mid\left\|g^{*}\right\|=1\right\}} \int_{\left\{\lambda \in \sigma \mid e^{2 s|\lambda|^{1 / \beta}} e^{t \operatorname{Re} \lambda>n\}}\right.} e^{s|\lambda|^{1 / \beta}} e^{t \operatorname{Re} \lambda} d v\left(f, g^{*}, \lambda\right) \longrightarrow 0 \quad \text { as } n \longrightarrow \infty .
$$

Indeed, as follows from the preceding argument, the specific choice of $s=t /[1+$ $\left.(2 / b)^{\beta}\right]^{1 / \beta}>0$ allows to partition the set $\left\{\lambda \in \sigma \mid e^{2 s|\lambda|^{1 / \beta}} e^{t \operatorname{Re} \lambda}>n\right\}$ into two subsets $\sigma_{1}$ and $\sigma_{2}$ in such a way that $\sigma_{1}$ is bounded and

$$
e^{s|\lambda|^{1 / \beta}} e^{t \operatorname{Re} \lambda}=1, \quad \lambda \in \sigma_{2} .
$$


Therefore,

$$
\begin{aligned}
& \sup _{\left\{g^{*} \in X^{*} \mid\left\|g^{*}\right\|=1\right\}} \int_{\left\{\lambda \in \sigma \mid e^{s|\lambda|^{1 / \beta}} e^{t \operatorname{Re} \lambda>n\}}\right.} e^{s|\lambda|^{1 / \beta}} e^{t \operatorname{Re} \lambda} d v\left(f, g^{*}, \lambda\right) \\
& \leq \sup _{\left\{g^{*} \in X^{*} \mid\left\|g^{*}\right\|=1\right\}} \int_{\left\{\lambda \in \sigma_{1} \mid e^{s|\lambda|^{1 / \beta}} e^{t \operatorname{Re} \lambda}>n\right\}} e^{s|\lambda|^{1 / \beta}} e^{t \operatorname{Re} \lambda} d v\left(f, g^{*}, \lambda\right) \\
& \quad+\sup _{\left\{g^{*} \in X^{*} \mid\left\|g^{*}\right\|=1\right\}} \int_{\left\{\lambda \in \sigma_{2} \mid e^{s|\lambda|^{1 / \beta}} e^{t \operatorname{Re} \lambda>n\}}\right.} e^{s|\lambda|^{1 / \beta}} e^{t \operatorname{Re} \lambda} d v\left(f, g^{*}, \lambda\right)
\end{aligned}
$$

(since $\sigma_{1}$ is bounded, there is such a $C>0$ that $e^{s|\lambda|^{1 / \beta}} e^{t \operatorname{Re} \lambda} \leq C, \lambda \in \sigma_{1}$; by (2.8))

$$
\begin{aligned}
& \leq \sup _{\left\{g^{*} \in X^{*} \mid\left\|g^{*}\right\|=1\right\}} C 4 M\left\|E_{A}\left(\left\{\lambda \in \sigma_{1} \mid e^{s|\lambda|^{1 / \beta}} e^{t \operatorname{Re} \lambda}>n\right\}\right) f\right\|\left\|g^{*}\right\| \\
& +\sup _{\left\{g^{*} \in X^{*} \mid\left\|g^{*}\right\|=1\right\}} 4 M\left\|E_{A}\left(\left\{\lambda \in \sigma_{2} \mid e^{s|\lambda|^{1 / \beta}} e^{t \operatorname{Re} \lambda}>n\right\}\right) f\right\|\left\|g^{*}\right\| \\
& \leq 4 C M\left\|E_{A}\left(\left\{\lambda \in \sigma_{1} \mid e^{s|\lambda|^{1 / \beta}} e^{t \operatorname{Re} \lambda}>n\right\}\right) f\right\|
\end{aligned}
$$$$
+4 M\left\|E_{A}\left(\left\{\lambda \in \sigma_{2} \mid e^{s|\lambda|^{1 / \beta}} e^{t \operatorname{Re} \lambda}>n\right\}\right) f\right\| \quad \text { (by the strong continuity of the s.m.) }
$$$$
\longrightarrow 0 \text { as } n \longrightarrow \infty \text {. }
$$

According to [23, Proposition 3.1], we have proved that, for any $f \in X$ and $t>0$,

$$
e^{t A} f \in D\left(e^{s|A|^{1 / \beta}}\right)
$$

where $s=t /\left[1+(2 / b)^{\beta}\right]^{1 / \beta}>0$.

Hence, for any $f \in X$,

$$
e^{t A} f \in \bigcup_{s>0} D\left(e^{s|A|^{1 / \beta}}\right) \subseteq \mathscr{E}^{\{\beta\}}(A), \quad 0<t<\infty .
$$

“ONLY IF” PART. We prove this part by contrapositive.

Assume the negation of "for some positive $b$ and real $a, \sigma(A) \subseteq\{\lambda \in \mathbb{C} \mid \operatorname{Re} \lambda \leq$ $\left.a-b|\operatorname{Im} \lambda|^{1 / \beta}\right\}$," that is, for any positive $b$ and real $a$, the set $\sigma(A) \backslash\{\lambda \in \mathbb{C} \mid \operatorname{Re} \lambda \leq$ $\left.a-b|\operatorname{Im} \lambda|^{1 / \beta}\right\} \neq \varnothing$. Whence it is easy to infer that, for any natural $n$, the set

$$
\sigma(A) \backslash\left\{\left.\lambda \in \mathbb{C}\left|\operatorname{Re} \lambda \leq-\frac{1}{n}\right| \operatorname{Im} \lambda\right|^{1 / \beta}\right\}
$$

is unbounded.

Hence, we can choose a sequence of points of the complex plane $\left\{\lambda_{n}\right\}_{n=1}^{\infty}$ in the following way:

$$
\begin{gathered}
\lambda_{n} \in \sigma(A), \quad n=1,2, \ldots, \\
\operatorname{Re} \lambda_{n}>-\frac{1}{n}|\operatorname{Im} \lambda|^{1 / \beta}, \quad n=1,2, \ldots, \\
\lambda_{0}:=0, \quad\left|\lambda_{n}\right|>\max \left[n,\left|\lambda_{n-1}\right|\right], \quad n=1,2, \ldots
\end{gathered}
$$


The latter, in particular, implies that the points $\lambda_{n}$ are distinct:

$$
\lambda_{i} \neq \lambda_{j}, \quad i \neq j
$$

Since the set

$$
\left\{\left.\lambda \in \mathbb{C}\left|\operatorname{Re} \lambda>-\frac{1}{n}\right| \operatorname{Im} \lambda\right|^{1 / \beta},|\lambda|>\max \left[n,\left|\lambda_{n-1}\right|\right]\right\}
$$

is open in $\mathbb{C}$ for any $n=1,2, \ldots$, there exists such a $\varepsilon_{n}>0$ that this set contains together with the point $\lambda_{n}$ the open disk centered at $\lambda_{n}$ :

$$
\Delta_{n}=\left\{\lambda \in \mathbb{C}|| \lambda-\lambda_{n} \mid<\varepsilon_{n}\right\}
$$

that is, for any $\lambda \in \Delta_{n}$,

$$
\begin{aligned}
& \operatorname{Re} \lambda>-\frac{1}{n}|\operatorname{Im} \lambda|^{1 / \beta}, \\
& |\lambda|>\max \left[n,\left|\lambda_{n-1}\right|\right] .
\end{aligned}
$$

Moreover, since the points $\lambda_{n}$ are distinct, we can regard that the radii of the disks, $\varepsilon_{n}$, are chosen to be small enough so that

$$
\begin{gathered}
0<\varepsilon_{n}<\frac{1}{n}, \quad n=1,2, \ldots, \\
\Delta_{i} \cap \Delta_{j}=\varnothing, \quad i \neq j \quad \text { (the disks are pairwise disjoint). }
\end{gathered}
$$

Note that, by the properties of the s.m., the latter implies that the subspaces $E_{A}\left(\Delta_{n}\right) X$, $n=1,2, \ldots$, are nontrivial since $\Delta_{n} \cap \sigma(A) \neq \varnothing$ and $\Delta_{n}$ is open, and

$$
E_{A}\left(\Delta_{i}\right) E_{A}\left(\Delta_{j}\right)=0, \quad i \neq j
$$

We can choose a unit vector $e_{n}$ in each subspace $E_{A}\left(\Delta_{n}\right) X$ and thereby obtain a vector sequence such that

$$
E_{A}\left(\Delta_{i}\right) e_{j}=\delta_{i j} e_{i}
$$

( $\delta_{i j}$ is the Kronecker delta symbol).

The latter, in particular, implies that the vectors $\left\{e_{1}, e_{2}, \ldots\right\}$ are linearly independent and that

$$
d_{n}:=\operatorname{dist}\left(e_{n}, \operatorname{span}\left(\left\{e_{k} \mid k \in \mathbb{N}, k \neq n\right\}\right)\right)>0, \quad n=1,2, \ldots
$$

Furthermore,

$$
d_{n} \nrightarrow 0 \text { as } n \longrightarrow \infty
$$


Indeed, if we assume the opposite: $d_{n} \rightarrow 0$ as $n \rightarrow \infty$, then, for any $n=1,2, \ldots$, we can find an $f_{n} \in \operatorname{span}\left(\left\{e_{k} \mid k \in \mathbb{N}, k \neq n\right\}\right)$ such that $\left\|e_{n}-f_{n}\right\|<d_{n}+1 / n$, which immediately implies that $e_{n}=E_{A}\left(\Delta_{n}\right)\left(e_{n}-f_{n}\right) \rightarrow 0$. Thus, such an assumption leads to a contradiction.

Therefore, there is a positive $\varepsilon$ such that

$$
d_{n} \geq \varepsilon, \quad n=1,2, \ldots
$$

As follows from the Hahn-Banach theorem, for each $n=1,2, \ldots$, there is an $e_{n}^{*} \in X^{*}$ such that

$$
\begin{gathered}
\left\|e_{n}^{*}\right\|=1, \\
\left\langle e_{i}, e_{j}^{*}\right\rangle=\delta_{i j} d_{i} .
\end{gathered}
$$

Let

$$
g^{*}:=\sum_{n=1}^{\infty} \frac{1}{n^{2}} e_{n}^{*}
$$

Hence,

$$
\begin{aligned}
\left\langle e_{n}, g^{*}\right\rangle & =\frac{d_{n}}{n^{2}} \quad(\text { by }(5.28)) \\
& \geq \frac{\varepsilon}{n^{2}} .
\end{aligned}
$$

Concerning the sequence of the real parts, $\left\{\operatorname{Re} \lambda_{n}\right\}_{n=1}^{\infty}$, there are two possibilities: it is either bounded or not. We consider separately each of them.

First, assume that the sequence $\left\{\operatorname{Re} \lambda_{n}\right\}_{n=1}^{\infty}$ is bounded, that is, there is such an $\omega>0$ that

$$
\left|\operatorname{Re} \lambda_{n}\right| \leq \omega, \quad n=1,2, \ldots
$$

Let

$$
f:=\sum_{n=1}^{\infty} \frac{1}{n^{2}} e_{n} .
$$

As can be easily deduced from (5.24),

$$
\begin{gathered}
E_{A}\left(\Delta_{n}\right) f=\frac{1}{n^{2}} e_{n}, \quad n=1,2, \ldots, \\
E_{A}\left(\bigcup_{n=1}^{\infty} \Delta_{n}\right) f=f .
\end{gathered}
$$

Also, for $n=1,2, \ldots$,

$$
\begin{aligned}
& v\left(f, g^{*}, \Delta_{n}\right) \geq\left|\left\langle E_{A}\left(\Delta_{n}\right) f, g^{*}\right\rangle\right| \quad(\text { by }(5.34)) \\
&=\left|\left\langle\frac{1}{n^{2}} e_{n}, g^{*}\right\rangle\right| \quad(\text { by }(5.31)) \\
&=\frac{d_{n}}{n^{4}} \geq \frac{\varepsilon}{n^{4}} .
\end{aligned}
$$


For an arbitrary $s>0$, we have

$$
\begin{aligned}
& \int_{\sigma(A)} e^{s|\lambda|^{1 / \beta}} e^{\operatorname{Re} \lambda} d v\left(f, g^{*}, \lambda\right) \quad(\text { by }(5.34)) \\
& =\int_{\sigma(A)} e^{s|\lambda|^{1 / \beta}} e^{\operatorname{Re} \lambda} d v\left(E_{A}\left(\bigcup_{n=1}^{\infty} \Delta_{n}\right) f, g^{*}, \lambda\right) \quad \text { (by the properties of the } \text { o.c.) } \\
& =\int_{\bigcup_{n=1}^{\infty} \Delta_{n}} e^{s|\lambda|^{1 / \beta}} e^{\operatorname{Re} \lambda} d v\left(f, g^{*}, \lambda\right) \\
& =\sum_{n=1}^{\infty} \int_{\Delta_{n}} e^{s|\lambda|^{1 / \beta}} e^{\operatorname{Re} \lambda} d v\left(f, g^{*}, \lambda\right) \\
& \quad\left(\text { for } \lambda \in \Delta_{n}, \text { by }(5.22),(5.32), \text { and }(5.23),|\lambda| \geq n, \text { and } \operatorname{Re} \lambda=\right. \\
& \geq \sum_{n=1}^{\infty} e^{s n^{1 / \beta}} e^{-(\omega+1)} v\left(f, g^{*}, \Delta_{n}\right) \quad(\text { by }(5.35)) \\
& \geq e^{-(\omega+1)} \sum_{n=1}^{\infty} \frac{\varepsilon e^{s n^{1 / \beta}}}{n^{4}}=\infty .
\end{aligned}
$$

This, by [23, Proposition 3.1], implies that

$$
e^{A} f \notin \bigcup_{t>0} D\left(e^{t|A|^{1 / \beta}}\right)
$$

Then, by (2.14), moreover,

$$
e^{A} f \notin \mathscr{E}^{\beta}(A)
$$

Hence, by Proposition 3.1, we infer that the orbit $e^{t A} f, t \geq 0$, does not belong to $\mathscr{E}^{\{\beta\}}((0, \infty), X)$.

Now, suppose that the sequence $\left\{\operatorname{Re} \lambda_{n}\right\}_{n=1}^{\infty}$ is unbounded. The sequence being bounded above, since $A$ generates a $C_{0}$-semigroup [11] (see also [22]), this means that there is a subsequence $\left\{\operatorname{Re} \lambda_{n(k)}\right\}_{k=1}^{\infty}$ such that

$$
\operatorname{Re} \lambda_{n(k)} \longrightarrow-\infty \text { as } k \longrightarrow \infty \text {. }
$$

Thus, without loss of generality, we can regard that

$$
\operatorname{Re} \lambda_{n(k)} \leq-k, \quad k=1,2, \ldots
$$

Consider the vector

$$
f:=\sum_{k=1}^{\infty} \frac{1}{k^{2}} e_{n(k)} .
$$


By (5.24),

$$
\begin{gathered}
E_{A}\left(\Delta_{n}(k)\right) f=\frac{1}{k} e_{n(k)}, \quad k=1,2, \ldots, \\
E_{A}\left(\bigcup_{k=1}^{\infty} \Delta_{n(k)}\right) f=f .
\end{gathered}
$$

For an arbitrary $s>0$, we similarly have

$$
\int_{\sigma(A)} e^{s|\lambda|^{1 / \beta}} e^{\operatorname{Re} \lambda} d v\left(f, g^{*}, \lambda\right)=\sum_{k=1}^{\infty} \int_{\Delta_{n(k)}} e^{s|\lambda|^{1 / \beta}} e^{\operatorname{Re} \lambda} d v\left(f, g^{*}, \lambda\right)=\infty .
$$

Indeed, for all $\lambda \in \Delta_{n(k)}$, based on (5.23), (5.40), and (5.22), we have

$$
\begin{aligned}
\operatorname{Re} \lambda=\operatorname{Re} \lambda_{n(k)}- & \left(\operatorname{Re} \lambda_{n(k)}-\operatorname{Re} \lambda\right) \leq \operatorname{Re} \lambda_{n(k)}+\left|\lambda_{n(k)}-\lambda\right| \\
\leq \operatorname{Re} \lambda_{n(k)}+ & \varepsilon_{n(k)} \leq-k+1 \leq 0 \\
& -\frac{1}{n(k)}|\operatorname{Im} \lambda|^{1 / \beta}<\operatorname{Re} \lambda .
\end{aligned}
$$

Therefore, for $\lambda \in \Delta_{n(k)}$,

$$
-\frac{1}{n(k)}|\operatorname{Im} \lambda|^{1 / \beta}<\operatorname{Re} \lambda \leq-k+1 \leq 0 .
$$

Whence, for $\lambda \in \Delta_{n(k)}$,

$$
\operatorname{Re} \lambda \leq-k+1 \leq 0, \quad|\lambda| \geq|\operatorname{Im} \lambda| \geq[n(k)(-\operatorname{Re} \lambda)]^{\beta} .
$$

Using these estimates, we have

$$
\begin{aligned}
\int_{\Delta_{n}(k)} & e^{s|\lambda|^{1 / \beta}} e^{\operatorname{Re} \lambda} d v\left(f, g^{*}, \lambda\right) \\
\geq & \int_{\Delta_{n(k)}} e^{[s n(k)-1](-\operatorname{Re} \lambda)} d v\left(f, g^{*}, \lambda\right) \\
& \quad\left(\text { for all } k^{\prime} \text { s sufficiently large so that } s n(k)-1>0 \text { and } k-1 \geq 1\right) \\
\geq & e^{[\operatorname{sn}(k)-1](k-1)} v\left(f, g^{*}, \Delta_{n(k)}\right) \quad(\text { by }(5.35)) \\
\geq & \frac{\varepsilon e^{[s n(k)-1]}}{n(k)^{4}} \longrightarrow \infty \quad \text { as } k \longrightarrow \infty .
\end{aligned}
$$

Similarly, we infer that the orbit $e^{t A} f, t \geq 0$, does not belong to the class $\mathscr{E}^{\{\beta\}}((0, \infty), X)$.

Having analyzed all the possibilities concerning $\left\{\operatorname{Re} \lambda_{n}\right\}_{n=1}^{\infty}$, we infer that the negation of "for some positive $b$ and real $a, \sigma(A) \subseteq\left\{\left.\lambda \in \mathbb{C}|\operatorname{Re} \lambda \leq a-b| \operatorname{Im} \lambda\right|^{1 / \beta}\right\}$ " implies that not every orbit of the $C_{0}$-semigroup $\left\{e^{t A} \mid t \geq 0\right\}$ belongs to the Gevrey class $\mathscr{E}^{\{}\{\beta\}((0, \infty), H)$, that is, $\left\{e^{t A} \mid t \geq 0\right\}$ is not an $\mathscr{E}^{\{\beta\}}$-semigroup.

Thus, the "only if" part has been proved by contrapositive. 
In particular, for $\beta=1$, we obtain a criterion of the generation of an analytic $C_{0^{-}}$ semigroup by a scalar type spectral operator [22] (see also [30]).

Observe that, for $0<\beta<1$, all the orbits of the $C_{0}$-semigroup $\left\{e^{t A} \mid t \geq 0\right\}$ are entire functions, which immediately implies that $A$ is bounded (see [20]).

6. A concluding remark. Similar results for a normal operator in a complex Hilbert space are discussed in a more general context in $[18,19]$ (see also [20, 21]).

\section{REFERENCES}

[1] J. M. Ball, Strongly continuous semigroups, weak solutions, and the variation of constants formula, Proc. Amer. Math. Soc. 63 (1977), no. 2, 370-373.

[2] N. Dunford, A survey of the theory of spectral operators, Bull. Amer. Math. Soc. 64 (1958), 217-274.

[3] N. Dunford and J. T. Schwartz, Linear Operators. Part I. General Theory, Pure and Applied Mathematics, vol. 7, Interscience Publishers, New York, 1958, with the assistance of W. G. Bade and R. G. Bartle.

[4] _ Linear Operators. Part II: Spectral Theory. Self Adjoint Operators In Hilbert Space, Interscience Publishers, New York, 1963, with the assistance of William G. Bade and Robert G. Bartle.

[5] _ Linear Operators. Part III: Spectral Operators, Interscience Publishers, New York, 1971.

[6] K.-J. Engel and R. Nagel, One-Parameter Semigroups for Linear Evolution Equations, Graduate Texts in Mathematics, vol. 194, Springer-Verlag, New York, 2000.

[7] M. Gevrey, Sur la nature analytique des solutions des équations aux dérivées partielles, Ann. Ec. Norm. Sup. Paris 35 (1918), 129-190 (French).

[8] S. Goldberg, Unbounded Linear Operators: Theory and Applications, McGraw-Hill, New York, 1966.

[9] J. A. Goldstein, Semigroups of Linear Operators and Applications, Oxford Mathematical Monographs, Clarendon Press, Oxford University Press, New York, 1985.

[10] R. Goodman, Analytic and entire vectors for representations of Lie groups, Trans. Amer. Math. Soc. 143 (1969), 55-76.

[11] E. Hille and R. S. Phillips, Functional Analysis and Semi-Groups, American Mathematical Society Colloquium Publications, vol. 31, American Mathematical Society, Rhode Island, 1957.

[12] H. Komatsu, Ultradistributions and hyperfunctions, Hyperfunctions and PseudoDifferential Equations (Proc. Conf. on the Theory of Hyperfunctions and Analytic Functionals and Applications, R. I. M. S., Kyoto Univ., Kyoto, 1971; Dedicated to the Memory of André Martineau), Lecture Notes in Math., vol. 287, Springer, Berlin, 1973, pp. 164-179.

[13] _ Ultradistributions. I. Structure theorems and a characterization, J. Fac. Sci. Univ. Tokyo Sect. IA Math. 20 (1973), 25-105.

[14] _ Microlocal analysis in Gevrey classes and in complex domains, Microlocal Analysis and Applications (Montecatini Terme, 1989), Lecture Notes in Math., vol. 1495, Springer-Verlag, Berlin, 1991, pp. 161-236.

[15] S. G. Krein and M. I. Khazan, Differential equations in a Banach space, Mathematical Analysis, Vol. 21, Itogi Nauki i Tekhniki, Akad. Nauk SSSR Vsesoyuz. Inst. Nauchn. i Tekhn. Inform., Moscow, 1983, pp. 130-264.

[16] S. Mandelbrojt, Series de Fourier et Classes Quasi-Analytiques de Fonctions, Gauthier-Villars, Paris, 1935.

[17] M. V. Markin, On the Carleman classes of vectors of a scalar type spectral operator, to appear in Int. J. Math. Math. Sci. 
[18]__ On the strong smoothness of weak solutions of an abstract evolution equation. I. Differentiability, Appl. Anal. 73 (1999), no. 3-4, 573-606.

[19] _ On the strong smoothness of weak solutions of an abstract evolution equation. II. Gevrey ultradifferentiability, Appl. Anal. 78 (2001), no. 1-2, 97-137.

[20] _ On the strong smoothness of weak solutions of an abstract evolution equation. III. Gevrey ultradifferentiability, Appl. Anal. 78 (2001), no. 1-2, 139-152.

[21]__ On the strong smoothness of weak solutions of an abstract evolution equation. IV. Carleman ultradifferentiability, to appear in Appl. Anal.

[22] _ A note on the spectral operators of scalar type and semigroups of bounded linear operators, Int. J. Math. Math. Sci. 32 (2002), no. 10, 635-640.

[23] _ On an abstract evolution equation with a spectral operator of scalar type, Int. J. Math. Math. Sci. 32 (2002), no. 9, 555-563.

[24] E. Nelson, Analytic vectors, Ann. of Math. (2) 70 (1959), 572-615.

[25] A. Pazy, On the differentiability and compactness of semigroups of linear operators, J. Math. Mech. 17 (1968), 1131-1141.

[26] _ Semigroups of Linear Operators and Applications to Partial Differential Equations, Applied Mathematical Sciences, vol. 44, Springer-Verlag, New York, 1983.

[27] A. Plesner, Spectral Theory of Linear Operators, Izdat. "Nauka", Moscow, 1965.

[28] V. V. Vasil'ev, S. G. Kreĭn, and S. I. Piskarëv, Operator semigroups, cosine operator functions, and linear differential equations, Mathematical Analysis, Vol. 28, Itogi Nauki i Tekhniki, Akad. Nauk SSSR Vsesoyuz. Inst. Nauchn. i Tekhn. Inform., Moscow, 1990, pp. 87-202.

[29] J. Wermer, Commuting spectral measures on Hilbert space, Pacific J. Math. 4 (1954), 355361.

[30] K. Yosida, On the differentiability of semigroups of linear operators, Proc. Japan Acad. 34 (1958), 337-340.

[31] _ Functional Analysis, Die Grundlehren der mathematischen Wissenschaften, vol. 123, Academic Press, New York, 1965.

Marat V. Markin: Department of Partial Differential Equations, Institute of Mathematics, National Academy of Sciences of Ukraine, 3 Tereshchekivs'ka Street, Kiev 01601, Ukraine

E-mail address: mmarkin@comcast.net 


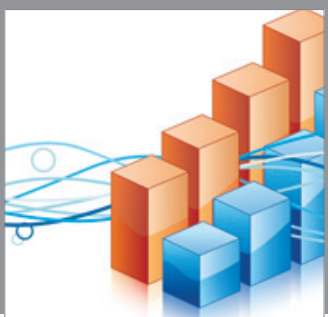

Advances in

Operations Research

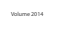

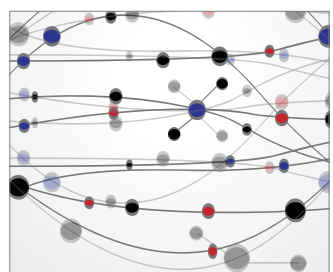

\section{The Scientific} World Journal
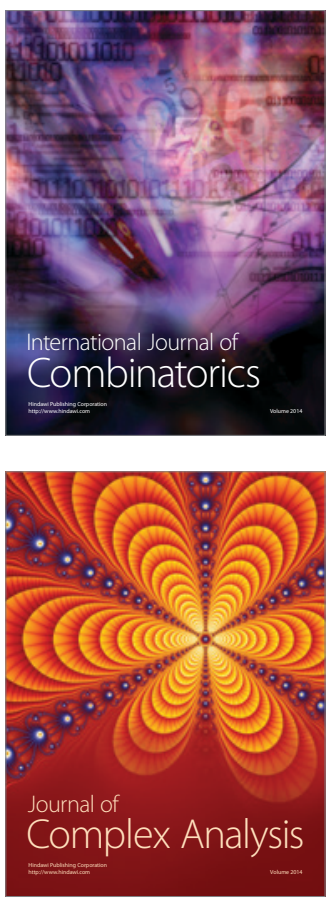

International Journal of

Mathematics and

Mathematical

Sciences
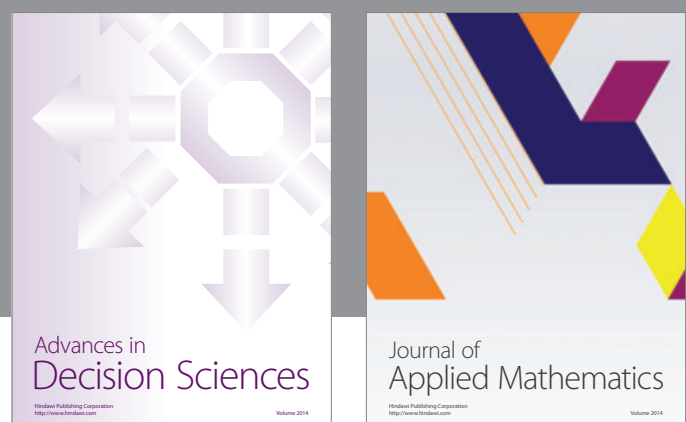

Journal of

Applied Mathematics
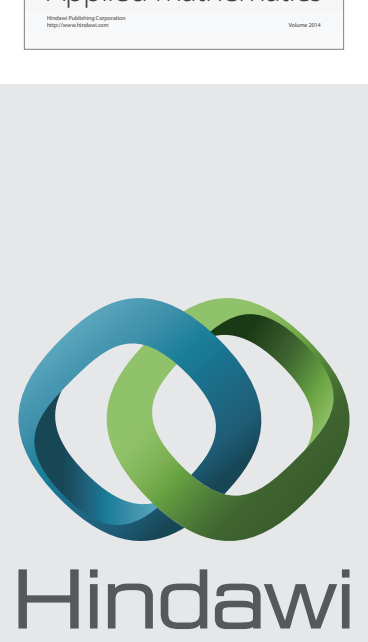

Submit your manuscripts at http://www.hindawi.com
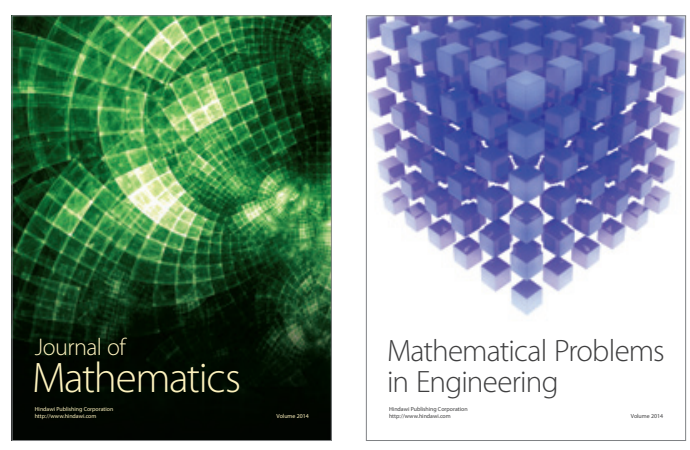

Mathematical Problems in Engineering
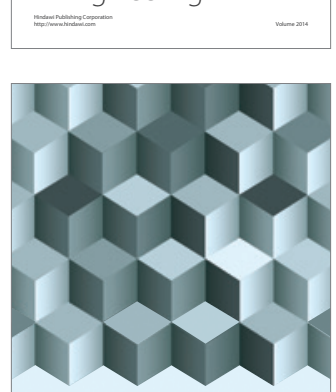

Journal of

Function Spaces
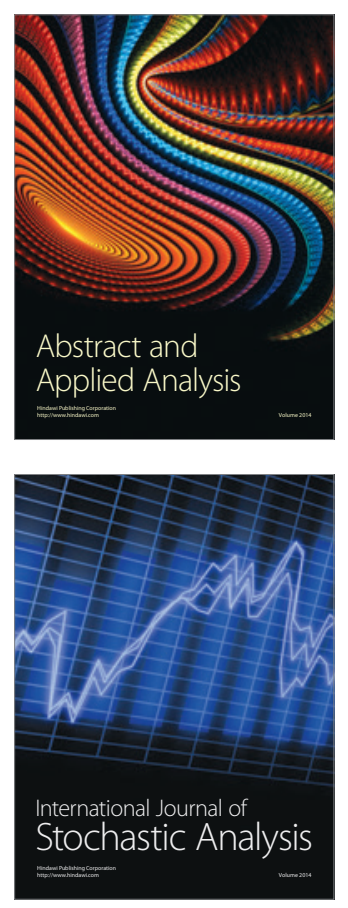

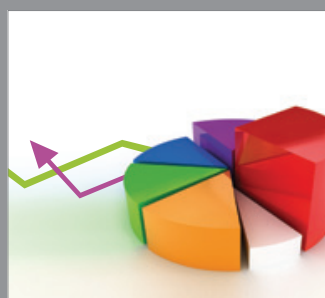

ournal of

Probability and Statistics

Promensencen
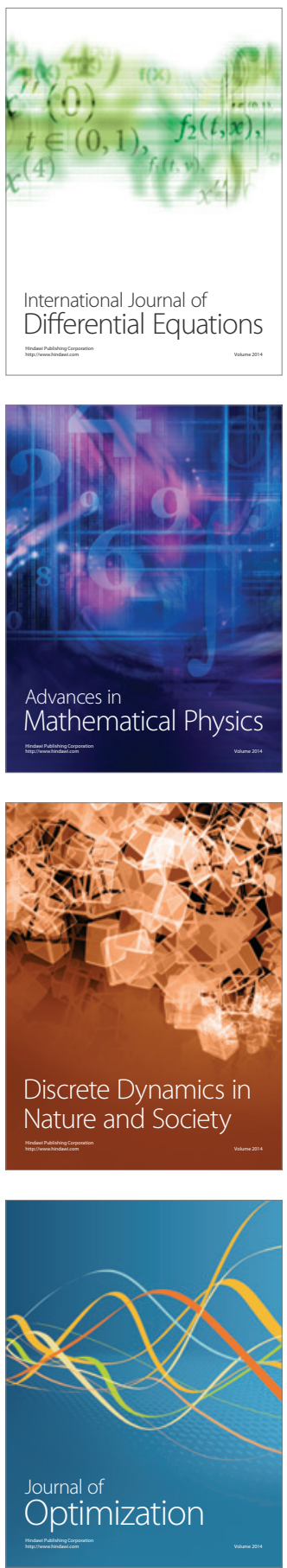